\title{
ECORS - Truc Vert 2008 : Qualification des modèles de houle et de morphodynamique
}

N. Sénéchal', F. Ardhuin², S. Abadie 3 , R. Almar', G. Arnaud ${ }^{3}$, M. Austin ${ }^{4}$, J.M. Barnoud ${ }^{5}$, E. Barthélémy ${ }^{5}$, C. Berni ${ }^{5}$, C. Blenkinsopp ${ }^{6}$, N. Bonneton ${ }^{1}$, P. $^{4}$ Bonneton $^{1}$, F. Bouée ${ }^{17}$, P. Bretel ${ }^{1}$, Jeff Brown ${ }^{5}$, Jena Brown ${ }^{5}$, S. Bujan ${ }^{1}$, D. Buscombe $^{4}$, S. Capo ${ }^{1}$, B. Castelle ${ }^{1}$, R. Certain ${ }^{7}$, F.X. Chassagneux ${ }^{5}$, G. Coco ${ }^{13}$, D. Corman ${ }^{2}$, C. Dallacosta ${ }^{5}$, A. Dehouck ${ }^{1}$, M. Delattre ${ }^{8}$, S. Delvallée ${ }^{8}$, S. de Vries $^{9}$, T. Dewez ${ }^{8}$, G. Dodet ${ }^{4}$, I. Emmanuel ${ }^{5}$, J.M. Escalier ${ }^{1}$, B. Francois ${ }^{8}$,

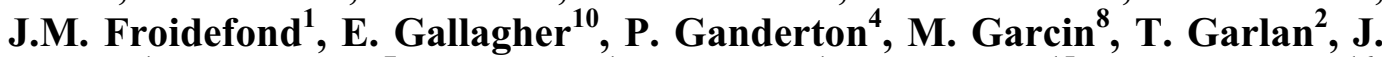
Gaunet $^{1}$, M. Gervais ${ }^{7}$, L. Gluard ${ }^{1}$, I. George ${ }^{1}$, F. Gouaud ${ }^{15}$, P. Grandjean ${ }^{16}$, F. Grasso ${ }^{5}$, R. Hampson ${ }^{5}$, V. Hanquiez ${ }^{1}$, M. Henriquez ${ }^{9}$, W. Hibberd ${ }^{4}$, D. Hurther $^{5}$, J.M. Lafosse ${ }^{17}$, M. Lagauzère ${ }^{5}$, A. Lambert, S. Lecacheux ${ }^{8}$, G. Le Cozannet $^{8}$, J. Le Drezigue ${ }^{15}$, F. Le Gall ${ }^{2}$, P. Lencou ${ }^{17}$, R. Le Roy ${ }^{2}$, A. Lusven ${ }^{2}$, J. Mac Mahan 11, R. Magne', V. Marieu ${ }^{1}$, H. Markies ${ }^{12}$, P. Marron ${ }^{3}$, N. Martiny $^{1}$, G. Masselink ${ }^{4}$, H. Michallet ${ }^{5}$, E. Mignot ${ }^{5}$, M. Minet ${ }^{1,2}, J_{\text {. Moon }}{ }^{4}, J_{\text {. }}$ Moreau $^{1}$,D. Morichon ${ }^{3}$, S. Morisset ${ }^{1}$, M. Mory ${ }^{3}$, A. Nahon ${ }^{1}$, J. Oman ${ }^{5}$, M. Outré $^{2}$, J.P. Parisot ${ }^{1}$, G. Payne ${ }^{13}$, R. Pedreros ${ }^{8}$, T. Poate ${ }^{4}$, A. Réjas ${ }^{1}$, A. Reniers $^{14}$, V. Rey ${ }^{15}$, E. Romieu ${ }^{8}$, P. Rouillé ${ }^{17}$, A. Ruiz De Alegria ${ }^{4}$, G. Ruessink $^{12}$, P. Russell ${ }^{4}$, M. Schippers ${ }^{9}$, M. Smit ${ }^{9}$, C. Sotin $^{1}$, D. Sous ${ }^{15}$, T. Stanton $^{11}$, J. Stockel ${ }^{11}$, J. Thiebaut ${ }^{8}$, E. Thornton ${ }^{11}$, J. Tinker ${ }^{4}$, M. Tissier ${ }^{1}$, I. Turner $^{6}$, B. Van Dam ${ }^{12}$, R. Vandromme ${ }^{8}$, M. Van Maarseveen ${ }^{12}$

${ }^{1}$ OASU-EPOC, Université Bordeaux I, avenue des Facultés 33405 Talence cedex n.senechal@epoc.u-bordeaux1.fr

${ }^{2}$ SHOM, 13 rue du Chatellier - CS 9280329228 Brest cedex 2 ardhuin@shom.fr

${ }^{3} \mathrm{LaSAGeC}^{2}$, Université Pau et Pays de l'Adour - France stephan.abadie@univ-pau.fr

${ }^{4}$ University of Plymouth - Royaume-Uni p.russell@plymouth.ac.uk

${ }^{5}$ LEGI, Université Joseph Fourier - Grenoble - France herve.michallet@hmg.inpg.fr

${ }^{6}$ University of New South Wales - Australie ian.turner@unsw.edu.au

${ }^{7}$ LEGEM, Université Perpignan - France certain@univ-perp.fr

${ }^{8}$ BRGM - France r.pedreros@brgm.fr

${ }^{9}$ Delft University of Technology - Pays Bas areniers@rsmas.miami.edu 


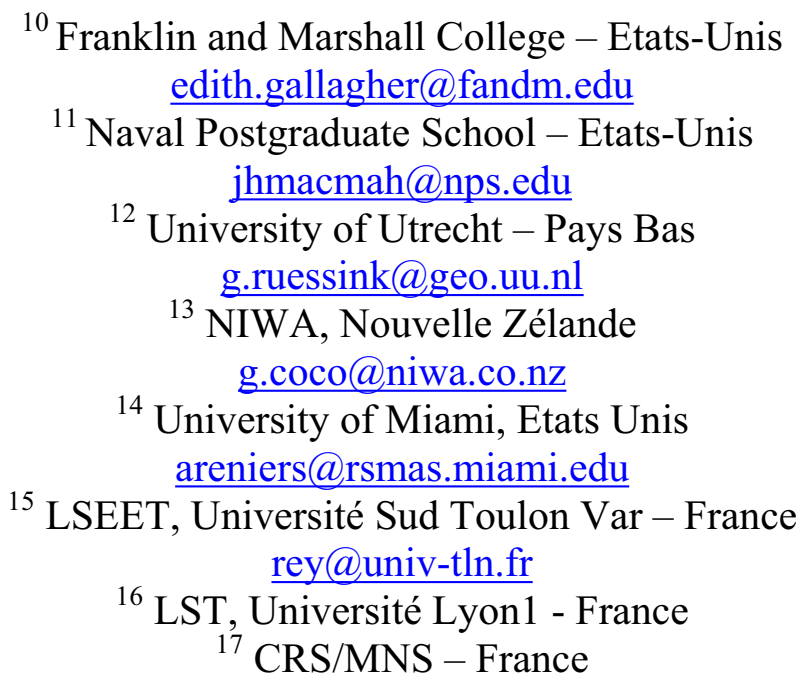

\title{
Résumé :
}

Cet article présente une campagne expérimentale internationale de grande envergure qui s'est déroulée en Mars-Avril 2008 sur la côte aquitaine. Cette campagne a permis de mettre en œuvre des instrumentations et méthodes innovantes afin de mesurer une vaste gamme de processus hydrodynamiques, sédimentaires et morphologiques en zone de surf et de jet de rive tout en couvrant des échelles temporelles de la milli secondes à plusieurs semaines et des échelles spatiales subcentimétriques à décamétriques. Cette campagne apportera un jeu de données unique avec des conditions énergétiques très fortes $(\mathrm{Hs}>5 \mathrm{~m})$ pour comprendre les processus mais également pour améliorer et valider les modèles numériques.

\begin{abstract}
:
The aim of this paper is to present a large international field experiment that took place in March-April 2008 on the French Aquitanian coast. During this field experiment, both very small and large spatial and temporal scales have been explored concerning hydrodynamic, sedimentary and morphodynamic processes in the surf and swash zones using innovative methods. This field experiment, one of the largest to take place in Europe (with the COAST 3D project), will provide a unique data set to explore surf zone and swash processes and improve numerical models in presence of very energetic events (5 storms with $\mathrm{Hs}>5 \mathrm{~m}$ ) on macrotidal multi-barred sandy beaches.
\end{abstract}

\section{Mots-clés :}

Expérimental - plage sableuse - méso, macrotidal -processus hydrodynamiques processus sédimentaires - morphodynamique - zones de surf et jet de rive 


\section{Introduction}

La dynamique des barres sableuses littorales, bien qu'ayant faite l'objet de nombreuses études ces dernières années, reste un phénomène encore difficilement modélisable de nos jours. Plusieurs explications peuvent être données à cela et parmi elles, le manque de données de terrain suffisamment complètes et précises pour d'une part observer et comprendre les phénomènes et d'autre part permettre d'améliorer et ensuite valider les modèles.

L'objectif de la campagne ECORS-TRUC VERT 2008 est justement de répondre à ces deux points, en ajoutant un jeu de données très énergétique et méso-tidal aux jeux de données existants. Initiée par le SHOM, la campagne est le fruit d'un partenariat porté par l'Université de Bordeaux I, avec de nombreux partenaires. Le site atelier choisi est la plage du Truc Vert, située au nord de la pointe du Cap Ferret et qui a déjà fait l'objet de plusieurs campagnes de terrain dans le cadre des programmes nationaux PNEC-ART 7 et PATOM. En effet, elle présente un système littoral complet incluant un double système de barres littorales (subtidal et intertidal), fortement tri-dimensionnelles et caractéristique de l'ensemble de la côte sableuse aquitaine, ainsi qu'un système dunaire complet comprenant une dune embryonnaire, une dune blanche et une dune grise. Plusieurs études (e. g. CASTELLE et al., 2007) ont permis de mettre en évidence le caractère dynamique de ces barres littorales soumises aux houles océaniques mais également de souligner les lacunes sur les données de terrain des précédentes campagnes afin de valider les modules de circulation, transport sédimentaire et de bathymétrie des modèles de morphodynamique.

En l'absence de moyens permanents de mesures en France, contrairement aux Etats-Unis, Pays-Bas ou Japon, aller au-delà des recherches précédentes a demandé une organisation particulière. Ainsi, la campagne ECORS-TRUC VERT 2008 est le résultat du travail collectif d'une centaine de personnes, enseignantchercheurs, chercheurs, ingénieurs, techniciens, officiers et officiers mariniers, post-doctorants, thésards, stagiaires et vacataires. Elle a réuni 16 équipes nationales et internationales de 6 pays : en France, EPOC, SHOM, LaSAGeC ${ }^{2}$, LEGEM, LEGI, LSEET, BRGM ; aux Pays Bas, l'Université d'Utrecht et l'université technologie de Delft; au Royaume Uni, l'Université de Plymouth ; en Australie, 1'Université de New South Wales ; en Nouvelle Zélande, la NIWA et aux Etats-Unis; La Naval Post-Graduate School, l'Université de Miami, 1'Université de Delaware et le Franklin and Marshall College.

Cette campagne a mobilisé d'importants moyens à la mer (bâtiment hydrographique Laplace et ses vedettes, jet skis), une infrastructure temporaire importante à terre, et le concours de Maîtres Nageurs Sauveteurs (MNS/CRS), mis à disposition par le Ministère de l'Intérieur. Elle s'est déroulée du 25 février 
au 18 Avril 2008 avec un pic d'activités «terrestres » entre le 3 Mars et le 12 Avril 2008.

Nous présentons ici l'ensemble de cette campagne, plus particulièrement l'originalité des solutions logistiques, les instruments et méthodes innovantes mis en œuvre pour étudier l'ensemble des processus hydro-sédimentaires.

\section{Logistique}

Le choix du site du Truc Vert (Aquitaine, Lège-Cap Ferret) éloigné de toute infrastructure humaine, a exigé la mise en place de l'ensemble de la logistique nécessaire à une telle campagne. Un groupe atelier logistique piloté par l'UMR EPOC a donc été mis spécialement en place afin de répondre aux exigences de cet environnement. Il s'agissait de mettre en place un campement sur la plage répondant aux besoins des équipes scientifiques: espace de vie suffisamment solide pour résister aux intempéries hivernales, zone de travail sur ordinateurs alimentée en courant stable, pour l'acquisition et le pré- traitement des données, aire de stockage des équipements, réserve de carburant, réserve en eau, mise en place d'un serveur temps, transport des équipements sur site et aménagement des accès. La mise en place de cette infrastructure s'est appuyée sur les compétences des entreprises locales et le soutien des collectivités et administrations (mairie du Lège Cap Ferret, ONF, Préfecture, Préfecture Maritime, Phares et Balises, SDIS). La figure 1a illustre le campement scientifique mis en place et la figure $1 \mathrm{~b}$ illustre les moyens mis en œuvre au déploiement de ce campement.

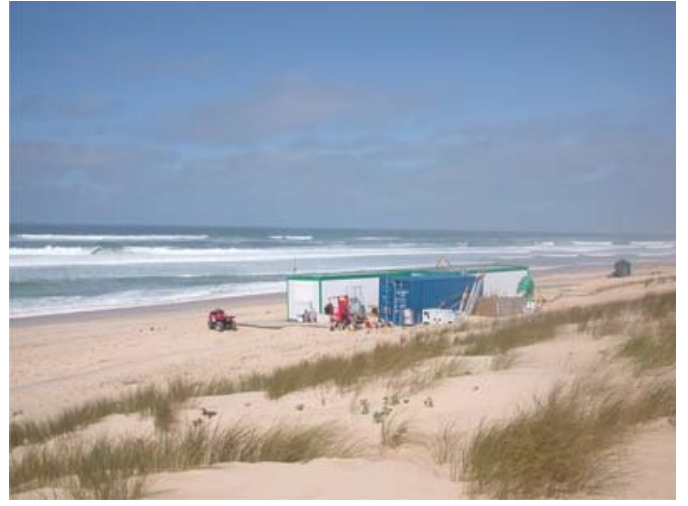

Figure 1a : campement scientifique déployé sur la dune embryonnaire.

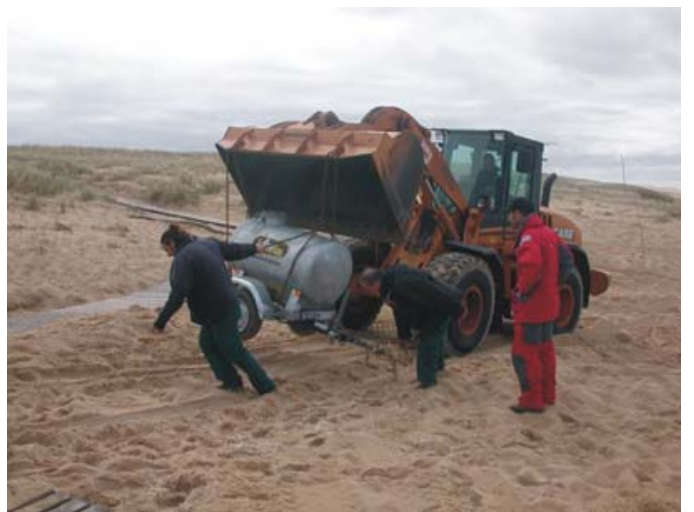

Figure $1 b$ : ravitaillement en eau douce.

\section{Les thématiques scientifiques abordées}

L'originalité de cette campagne tient dans la gamme d'échelles spatiotemporelles explorées par l'ensemble des partenaires: des phénomènes sub- 
centimétrique de couche limite jusqu'à l'évolution des bancs sableux dont les tailles caractéristiques sont de l'ordre de la centaine de mètres, et couvrant des échelles temporelles de l'ordre du centième de seconde à 6 semaines. Dans ce paragraphe nous allons présenter les différentes thématiques scientifiques abordées ainsi que les moyens mis en œuvre.

\subsection{Caractérisation des états de mer}

Un des préalables des campagnes de mesures de terrain est la connaissance mais également la prévision à plusieurs jours des états de mer. Il est en effet indispensable de connaître le forçage hydrodynamique sur la zone d'étude afin de mieux analyser l'ensemble des phénomènes qu'on y mesure mais également afin d'anticiper l'ensemble des opérations (levés hydrographiques et topographiques, déploiement et relèvement d'instruments, etc...) en fonction de l'évolution des conditions. Ces données sont également indispensables en vue de valider les modèles de morphodynamique puisqu'elles permettent de fixer les conditions limites de forçage de ces modèles numériques. Afin de répondre à ces besoins, deux bouées directionnelles Datawell ont été déployées par $55 \mathrm{~m}$ et $20 \mathrm{~m}$ de fond par le SHOM au large de la zone d'étude. Les données du houlographe le plus proche étaient envoyées en temps réel à une station de réception déployée sur le site, tandis que le second continue à alimenter la base de données CANDHIS du CETMEF. Ces mesures étaient complétées par des prévisions (http://www.previmer.org/previsions/vagues) générées deux fois par jour pour la zone d'étude avec une échéance maximale de 6 jours (MAGNE \& ARDHUIN 2008).

Parallèlement, le BRGM a déployé un système d'appareils photographiques à partir duquel il développe une méthode de détermination de l'altitude du plan d'eau et de la hauteur des vagues à la côte en s'appuyant sur la reconstruction du relief par stéréoscopie. Ce système est actuellement en cours de développement et les données de cette campagne permettront de le valider prochainement.

\subsection{Suivi de l'évolution de la morphologie de la plage}

L'objectif de cette thématique est de mesurer les réponses de la plage à l'échelle morphologique, en particulier de suivre l'évolution du volume sableux de la plage, la position, la forme et l'amplitude des barres sableuses, l'orientation des chenaux, la dynamique de la berme et du pied de dune. Cet atelier topographie a pu être mené à bien efficacement grâce à la mutualisation des moyens EPOC, BRGM, LEGEM, SHOM et NPS ainsi que le concours des MNS/CRS. Plusieurs méthodes ont été couplées afin de réaliser une topographie/bathymétrie aussi complète que possible à chaque marée basse diurne. La surface ainsi couverte 
représente une zone couvrant l'ensemble de la plage intertidale sur une bande littorale de $3 \mathrm{kms}$ et couvrant ponctuellement la zone subtidale jusqu'à une distance de $1,5 \mathrm{kms}$ de la côte. La figure 2 représente un exemple de levés réalisés par l'atelier topographie avec les différentes méthodes mises en œuvre.

La première méthode a consisté à des relevés GPS cinématique. En fonction de l'accessibilité de la zone, ces relevés se sont faits à pied, en quad (PARISOT et al. 2007) ou dans l'eau avec un GPS étanche (NPS). Plus au large, ces relevés GPS ont été couplés à des données de sondeurs, les mesures étant réalisées soit à partir d'un jet ski (EPOC, NPS) soit à partir de vedettes petits fonds (SHOM). Occasionnellement, ces mesures ont été couplées à des mesures théodolite effectuées en bas de plage au niveau des chenaux de vidange et des bancs sableux.

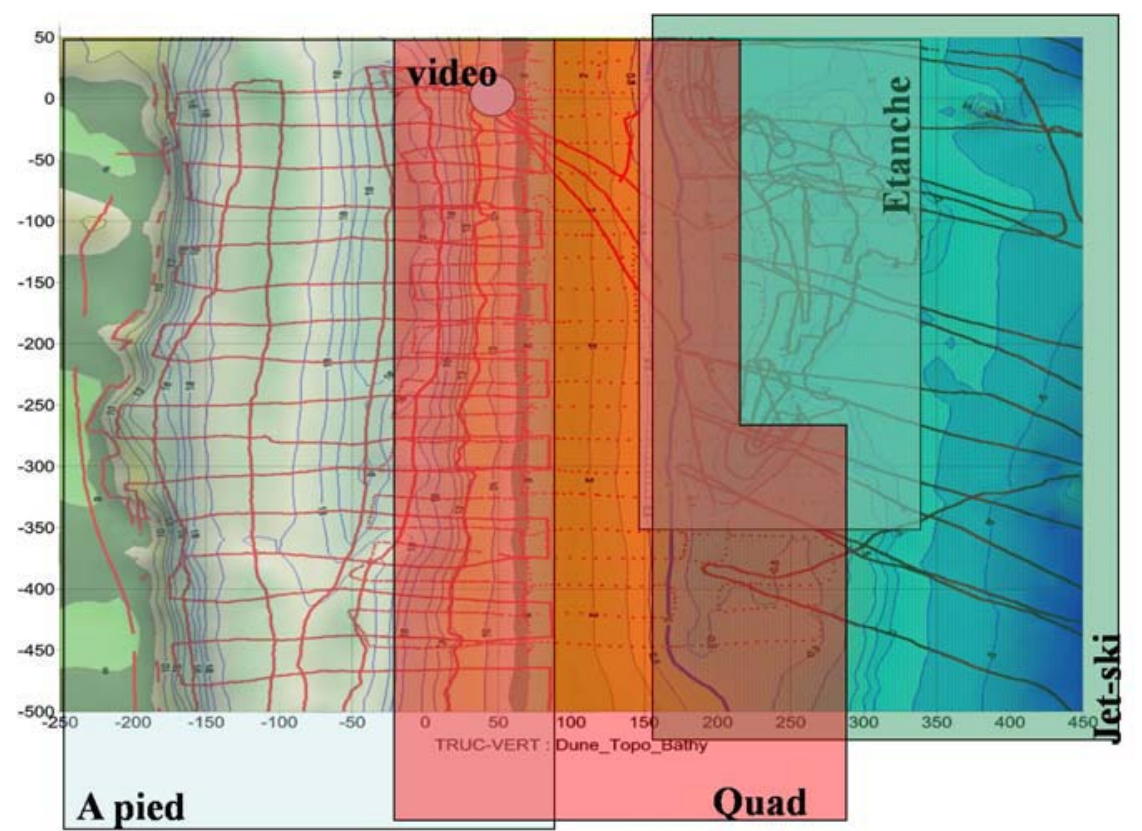

Figure 2 : exemples de relevés de la zone en emboîtant les différentes méthodes, les traits continus et pointillés représentent les mesures.

La deuxième méthode fait appel à des mesures indirectes : vidéo (EPOC, NIWA, University of Miami), satellitale (EPOC) ou aéroportée (Université Lyon 1 / Université Bretagne Occidentale). Un système de deux caméras a été déployé tout au long de la campagne à partir d'un échafaudage monté sur la dune littorale (figure 3a). Il permettra d'étudier la réponse morphologique de la plage sur un linéaire côtier de près de $2 \mathrm{kms}$ au large : qualitativement en s'appuyant sur des images moyennées qui mettent en évidence les barres sableuses immergées sur lesquelles les vagues déferlent préférentiellement (figure 3b) puis 
quantitativement en s'appuyant sur l'estimation de la vitesse des vagues ou la détection de la ligne d'eau (ALMAR et al., 2008). L'outil satellital (EPOC) s'appuie quant à lui sur la réfléctance de l'eau dans les canaux du bleu et/ou du vert. Ces réflectances permettent d'une part d'obtenir les produits «couleur de l'eau » (concentrations des divers constituants océaniques) par le biais d'algorithmes spécifiques (ex, FROIDEFOND et al., 1993); d'autre part d'estimer la bathymétrie, à partir d'une méthode développée au cours de la thèse de LAFON et al. (2002b) appliquée dans les passes du Bassin d'Arcachon puis adaptée à l'étude de la dynamique 2DH des barres sableuses littorales (LAFON et al., 2002a). Lors de cette campagne, des mesures de réflectances (à partir de spectroradiomètres) ainsi que des prélèvements d'eau (pour déterminer les concentrations en matières en suspension et en chlorophylle) ont été réalisées en jet-ski afin de tester une méthode de validation des algorithmes «couleur de l'eau » en zone côtière, plus complexe que l'océan ouvert d'un point de vue optique (MARTINY, 2002). Les mesures de réflectances réalisées en jet ski adjointes à des mesures de réflectance réalisées sur la plage permettront également de tester une méthode de calibration des algorithmes de calcul de la profondeur.

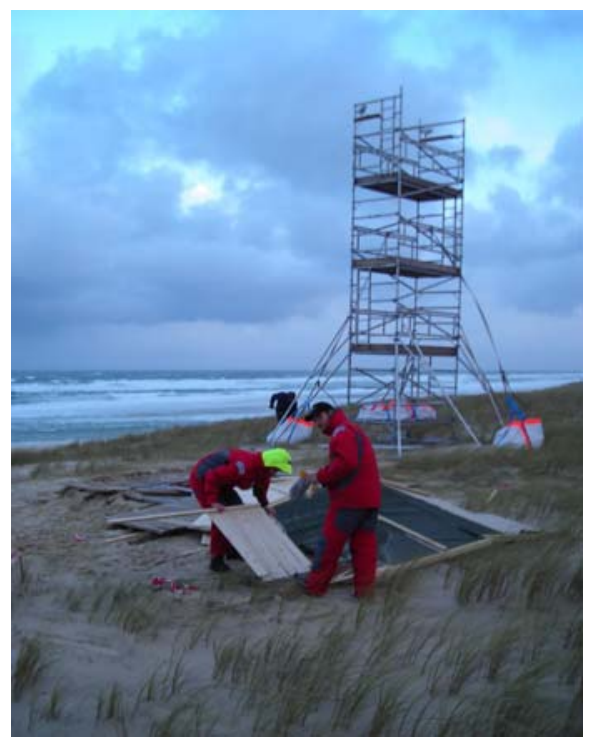

Figure 3a: système vidéo après la première tempête (EPOC-NIWA).

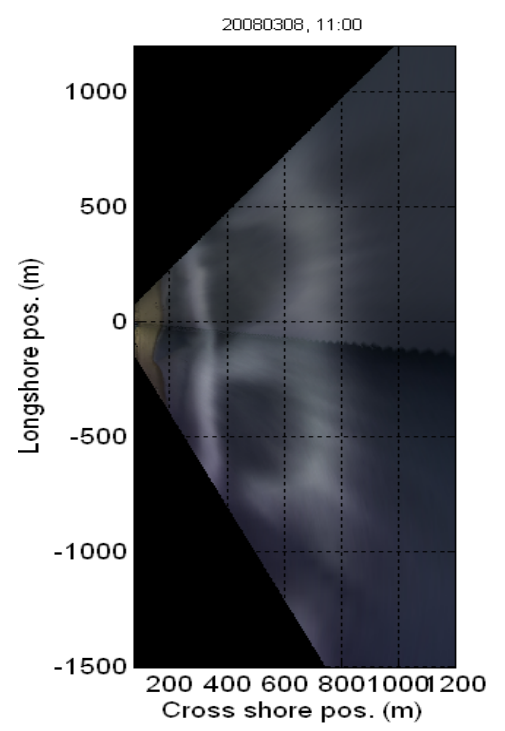

Figure $3 b$ : images moyennées révélant la forme en croissant des barres pré-littorales (EPOC). 
Enfin les méthodes aéroportées s'appuient sur des couples d'images et visent à reconstruire la topographie par stéréoscopie. Elles se développent dans le cadre du programme RELIEFS-AéroCoast3D financé par l'INSU.

\subsection{Caractérisation sédimentaire de la zone}

Cet atelier a réuni 6 laboratoires dont le SHOM, EPOC, LEGI, LSEET, $\mathrm{LaSAGeC}^{2}$ ainsi que le Franklin and Marshall College. L'un des objectifs de cet atelier est de mettre en place une cartographie de la granulométrie de surface des sédiments en vue d'établir une relation entre celle-ci et les structures morphologiques mais également d'en étudier la variation dans l'espace 2D et dans le temps. L'autre objectif de cet atelier est d'étudier la compacité de la plage en fonction de la marée et de la houle mais également d'étudier la porosité locale du sédiment. La variation de cette porosité sous l'influence des vagues est un élément intéressant dans un contexte de connaissance des processus d'érosion. Parfois, en effet, des couches entières de sol semblent avoir un comportement particulier en porosité avant une érosion soudaine de type liquéfaction.

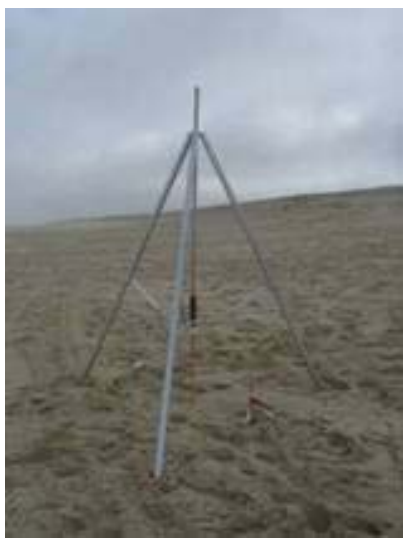

Figure 4a:

pénétromètre dynamique (EPOC)

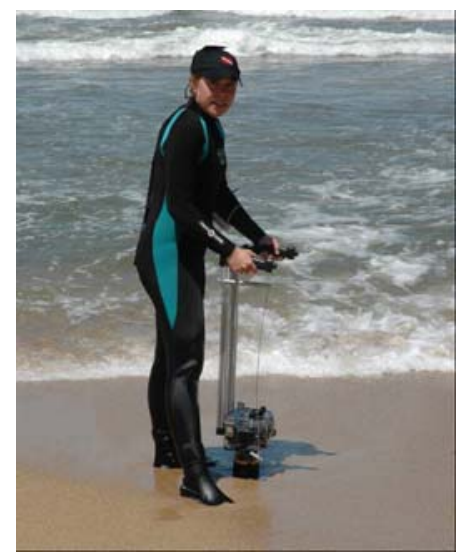

figure $4 b$ : prise de vue haute résolution (Franklin and Marshall college)

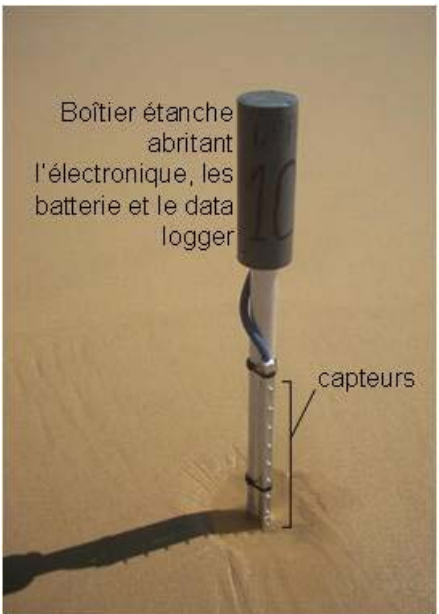

Figure 4c: perches résistives (LaSAGeC ${ }^{2}$ )

Plusieurs méthodes ont été mises en œuvre durant cette campagne afin de répondre à ces questions. En particulier, trois campagnes de prélèvements ont été réalisées (SHOM, EPOC), couvrant l'ensemble de la zone intertidale sur un linéaire côtier d'environ $600 \mathrm{~m}$ avec un maillage en $\mathrm{X}$ et en $\mathrm{Y}$ uniforme de $25 \mathrm{~m}$, 
chaque prélèvement étant localisé par GPS et associé à une prise de vue photographique. Les échantillons seront ensuite analysés en laboratoire par mircogranulométrie laser et tamisage de la fraction grossière (SHOM). Parallèlement à ces campagnes de prélèvements, la compacité de la plage a été analysée sur deux profils à partir de mesures de pénétrabilité dynamique (figure 4a). Des relevés sédimentaires ont également été effectués sur une profondeur correspondant à la longueur d'enfoncement de la tige du pénétromètre (EPOC). Journellement des prises de vues à très haute résolution ont également été effectuées dans la zone intertidale, qui ont pour but de réaliser une classification granulométrique par analyse d'image (figure 4b). Enfin des perches résistives $\left(\mathrm{LaSAGeC}^{2}\right)$ ont été déployées le long de profils perpendiculaires au trait de côte (figure 4c). Lorsque le sol est saturé, elles permettent d'obtenir une information sur la porosité locale du sédiment. Les données de ces perches sont couplées à la mesure locale de l'hydrodynamique.

\subsection{Processus turbulents et dynamique sédimentaire}

L'objectif de ce thème est de caractériser la stabilité du substrat sédimentaire et d'évaluer les flux sédimentaires instantanés couplés à des mesures hydrodynamiques à très hautes fréquences. L'un des objectifs est d'identifier et quantifier à partir des mesures in situ les processus et mécanismes fins hydrosédimentaires dominant les flux cross-shore de sable à l'échelle des vagues et du cycle de marée pour différents niveaux de forçage. Le but à long terme est de quantifier par la mesure sous les vagues, de la pression, la vitesse, la concentration en sable, et la position du fond, les effets tels que le déphasage temporel entre les vitesses orbitale et en couche limite de fond, l'asymétrie locale de la houle et l'accélération associée, le courant de retour autour du point de déferlement, la mise en suspension liée au déferlement et / ou à la propagation du ressaut. A ce jour, les différents modèles de débit solide tiennent compte de ces effets sous forme paramétrée sans connaître au préalable les contributions relatives des différents mécanismes précédents. Pour étudier la stabilité du substrat sédimentaire, plusieurs approches ont été utilisées. Un des premières approches a consisté à développer des capteurs optiques et résistifs pour localiser l'interface eau/sédiment, estimer la porosité du lit et l'apparition de bouffées de sédiments en suspension dans la colonne d'eau. Des études récentes, menées dans le cadre du projet européen LIMAS (Liquefaction Around Marine Structures, $V^{\mathrm{e}}$ PCRD), ont mis en évidence la variabilité de la transmission dans le sol des pressions induites par les vagues (PIEDRA-CUEVA et al., 2005). Ces effets fortement non-linéaires peuvent conduire à la déstabilisation complète d'une couche de sable (BONJEAN et al., 2004 ; MORY et al., 2004). 


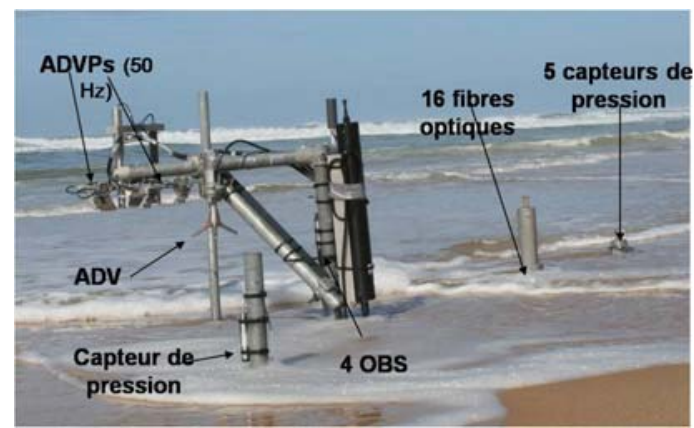

Figure 5a : système de fibres optiques et ADVP (LEGI)

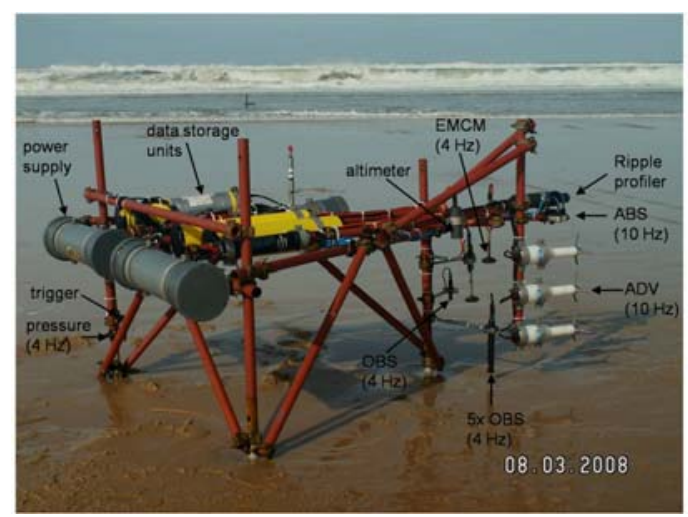

Figure 5c: structure de l'université d'Utrecht.

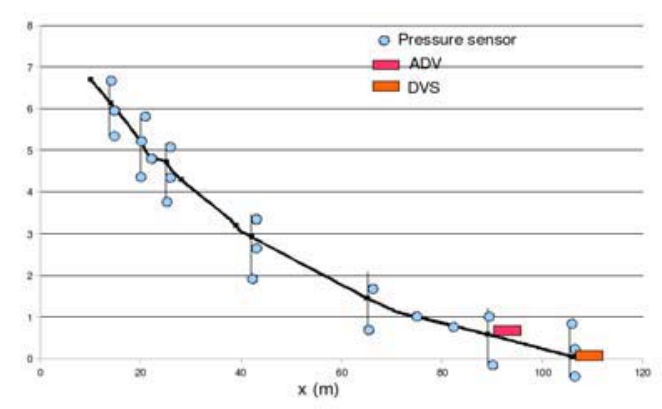

figure $5 b$ : ligne de capteurs de pression disposés au-dessus et sous le sable (LSEET).

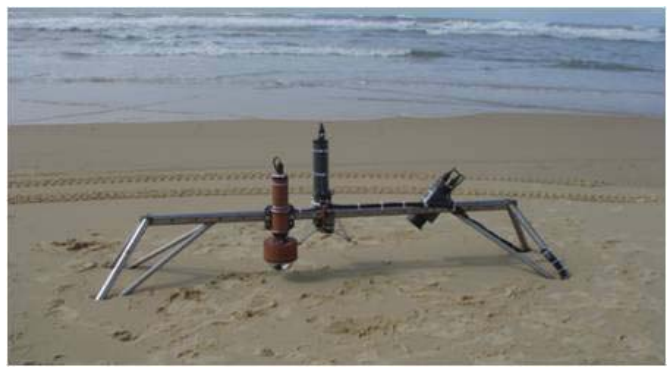

Figure 5d: instrumentation de la NPS

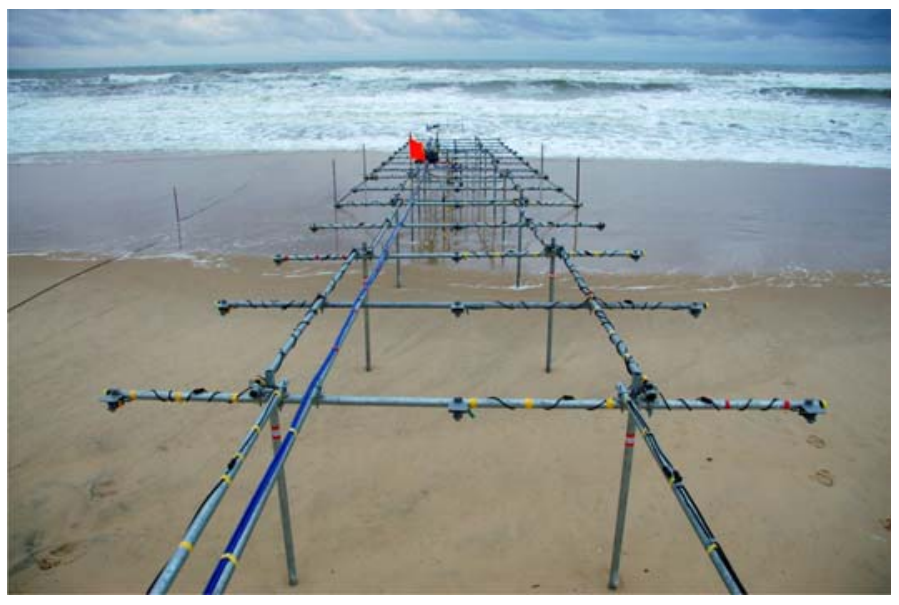

Figure 5e : structure Universités Plymouth et New South Wales 
Le suivi du niveau de sol et de sa stabilité a été rendu possible grâce au développement au LEGI d'un appareil de mesures par fibres optiques (figure 5a, MICHALLET et al., 2005). Ce système est constitué de plusieurs capteurs de type FOBS (Fiber Optics Backscatter Sensors). Il permet d'estimer les concentrations en suspension dans la colonne d'eau, le niveau du lit ainsi que la mobilité des grains de sable à l'intérieur du sol. Couplé à la mesure du forçage et des variations de pression dans le sol, il permet de déterminer les conditions d'érosion et de mise en suspension des sédiments. Ces mesures ont été associées aux mesures de vitesse turbulente dans la colonne d'eau afin d'obtenir une estimation la plus complète possible du flux sédimentaire. Parallèlement le réseau de "perches résistives" développées par le $\mathrm{LaSAGeC} 2$ et l'entreprise IMARTEC a été déployé suivant plusieurs profils dans la zone intertidale (figure $4 \mathrm{c}$ ). Les perches permettent dans un premier temps de situer à tout instant la position de l'interface eau/sédiment avec une résolution spatiale de $0,05 \mathrm{~m}$ et ce quelle que soit la dynamique extérieure (zone de surf, swash, ...). La connaissance des volumes de sable localement érodés permettra dans un second temps d'évaluer les flux instantanés de sédiments et de déterminer les éléments extérieurs qui favorisent ces flux. Enfin, le LSEET a déployé une ligne transversale de capteurs de pression (figure 5b), disposés au dessus et dans le sable afin notamment d'étudier les variations de pression induites dans le sable ainsi que les mouvements de la nappe phréatique associés à la marée. Ces données couplées aux relevés topographiques et à des données hydrodynamiques devraient permettre de mieux comprendre les relations entre d'une part les transformations non linéaires des vagues (génération des harmoniques et sous-harmoniques) et la réponse de l'interface eau/sédiment.

Concernant la quantification des flux sédimentaires, plusieurs méthodes ont également été utilisées. La première méthode s'est appuyée sur un couplage de données hydrodynamiques à très hautes fréquences avec des données de concentration en sédiment. Ces mesures ont notamment été possibles par le développement de Profileurs Ultrasonores de Flux de Particules (ADVP). Cette méthode permet de compléter les mesures de vitesse, de pression et de concentration, par des mesures haute-résolution de profils des 3 composantes de la vitesse et de concentration sédimentaire (figure 5a). L'échantillonnage simultané et quasi-instantané des profils de vitesse et de concentration par un ADVP se fait dans les mêmes volumes de mesure et à des fréquences d'acquisition permettant la résolution des échelles infra-gravitaires (100s) et inertielles turbulentes. L'atout majeur de cet outil par rapport aux instruments commerciaux existants (ADCP, $\mathrm{ADV}, \mathrm{OBS}, \mathrm{CTD}, \mathrm{ABS})$ réside dans sa capacité à estimer les vitesses (HURTHER et al. 2007a, 2007b) et les flux sédimentaires en suspension 
(HURTHER et LEMMIN 2003) à haute résolution spatiale et temporelle dans la couche limite de fond. Parallèlement l'Université d'Utrecht, la NPS et les Universités de Plymouth et New South Wales ont développé des instrumentations qui associent le couplage des mesures de vitesse et de concentration sédimentaire avec des suivis très hautes fréquences de l'interface eau / sédiments, en identifiant notamment la présence ou non de rides. Si les universités d'Utrecht et de la NPS (figure $5 \mathrm{c}$ et $5 \mathrm{~d}$ ) ont exploré l'ensemble des dynamiques extérieures (jet de rive, zone de surf....) en un point fixe, les universités de Plymouth et de New South Wales se sont concentrées sur la dynamique dans la zone de jet de rive sur une ligne transversale (figure 5e). Ces derniers ont déployé une structure longue de $28 \mathrm{~m}$ et large de $4 \mathrm{~m}$ composée de 45 sondeurs ultrasoniques positionnés au dessus du niveau moyen de marée haute. Entre ces sondeurs, cinq stations mesuraient les vitesses, pressions et concentrations en sédiments, permettant ainsi de suivre l'évolution du fond à l'échelle d'une vague mais également de mesurer les gradients dans l'hydrodynamique locale.

\subsection{Ondes de surface}

La dynamique des vagues en milieu littoral et particulièrement en zone de surf a beaucoup progressé ces dernières années. Par exemple, le mécanisme « depthinduced-breaking " a été mesuré en milieu naturel et analysé en détail (Raubenheimer et al. (1996); Sénéchal et al. (2001)). Si notre connaissance et notre capacité à modéliser la structure spatiale des quantités moyennes de la houle sont bonnes, en revanche il est nécessaire de progresser dans la compréhension de plusieurs phénomènes ondulatoires instationnaires qui jouent un rôle important dans la dynamique des plages, notamment ondes longues ou ondes infragravitaires et cinématique des fronts d'onde déferlée.

Si l'énergie contenue dans la bande infragravitaire est très faible par rapport à l'énergie véhiculée par les vagues au large, dans la zone de surf et près de la ligne d'eau, cette tendance peut rapidement s'inverser. De plus en plus d'études s'intéressent au rôle de ces ondes dans la mise en suspension et le transport des sédiments, en particulier dans la zone de jet de rive où le spectre est souvent dominé par ce que l'on appelle le «surf beat». Les mécanismes de génération de ces ondes infragravitaires (libération d'ondes liées, variations du point de déferlement...) ne sont pas encore à ce jour clairement identifiés et l'approche in situ reste encore très difficile car sa mise en œuvre est très complexe.

Sur les plages de pente douce à modérée comme celles rencontrées sur la côte Aquitaine, il est couramment admis que la réflexion des vagues est faible et peut être négligée alors qu'au contraire les ondes longues sont totalement réfléchies. A ce jour aucune mesure in situ ne permet en réalité de valider ces hypothèses. $\mathrm{Ce}$ 
point parait primordial compte tenu qu'un grand nombre de théories (ondes de bord, ondes stationnaires....) s'appuie sur cette hypothèse. Les résultats de cet objectif permettraient également de préciser les conditions limites (au niveau de la ligne d'eau) pour les modèles hydrodynamiques.
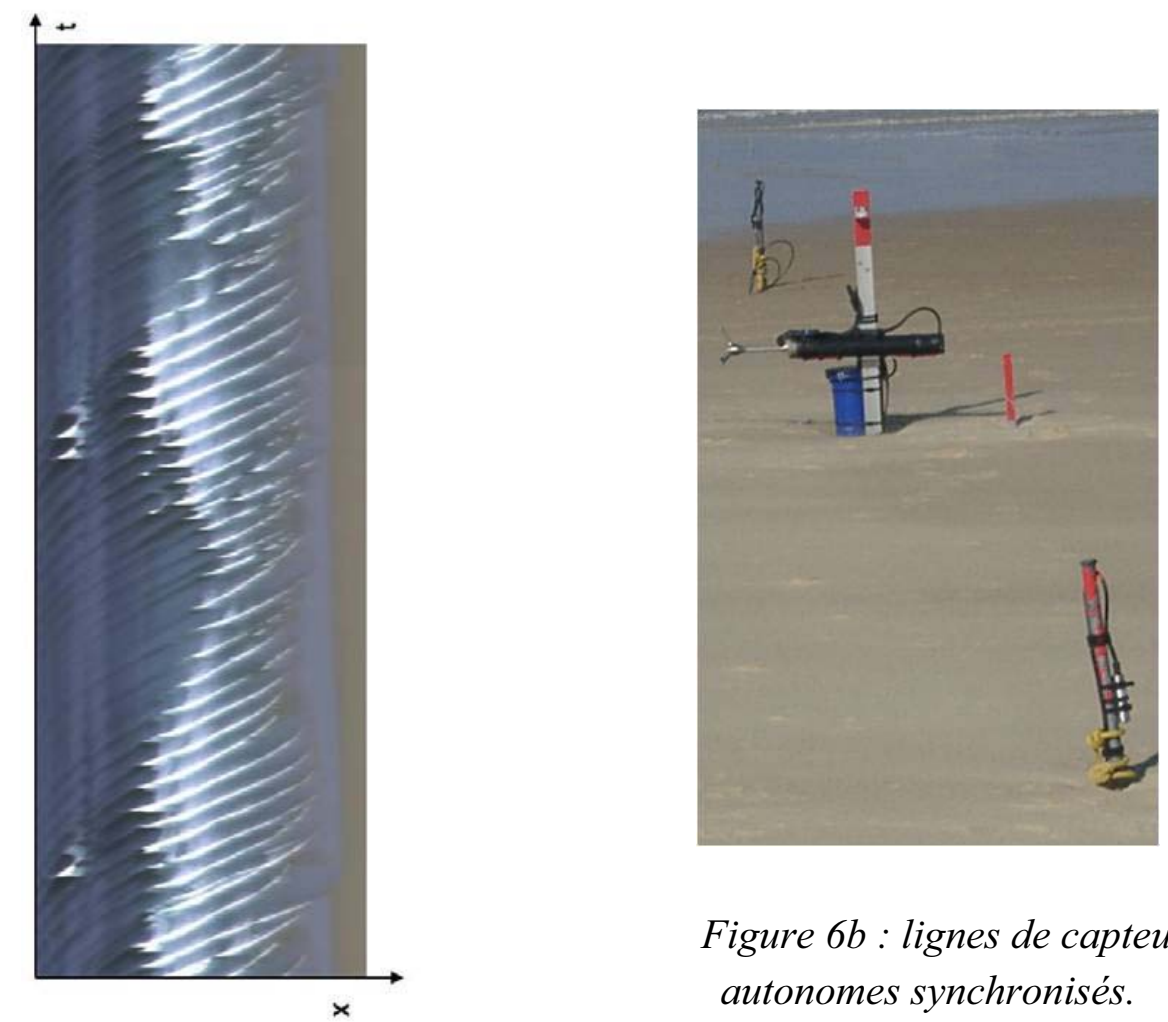

Figure $6 b$ : lignes de capteurs autonomes synchronisés.

Figure 6a: Timestacks

correspondant à l'évolution temporelle

d'une ligne de pixels.

De nombreuses théories de construction des barres prélittorales et de compréhension de leur rythmicité le long de nos côtes s'appuient sur la structure des ondes longues dans la zone de surf: ondes de bord et ondes stationnaires plus particulièrement. L'idée ici serait d'approfondir notre connaissance sur la structure spatio-temporelle de ces ondes en particulier en présence d'une bathymétrie complexe (systèmes de barres-baïnes) et d'une hydrodynamique énergétique (courant de baïne, courant de refente, dérive littorale) afin d'évaluer également les distorsions éventuelles de ces ondes dans ce contexte énergétique. Ces aspects « ondes infragavitaires » vont être analysés avec plusieurs approches. La NPS a déployé un réseau PUV parallèlement à la côte au niveau de la laisse de basse mer pour des vives eaux (isobathe $-1 \mathrm{~m}$ ) dont les caractéristiques spatiales 
ont été déterminées à partir de la structure probable des ondes de coin (« edge waves »). Les informations de cette ligne de capteurs pourront être couplées aux images vidéo des caméras qui ont filmé en continu avec une fréquence d'échantillonnage de $2 \mathrm{~Hz}$. La ligne transversale déployée par le LSEET permettra quant à elle d'évaluer la réflexion/dissipation des ondes courtes et longues sur une plage à pente douce mais aussi d'évaluer la distorsion des ondes de bord et stationnaires. La vidéo permettra également de suivre l'évolution de la ligne d'eau et d'explorer la dynamique du « surf beat » en zone de jet de rive.

Enfin, un autre point qui sera abordé dans ce thème concerne la dynamique des fronts d'onde déferlée. La cinématique des vagues en zones de surf et de jet de rive a été jusqu'à présent très peu étudiée que ce soit en laboratoire ou in situ. Or, la connaissance de la célérité des vagues déferlées $C_{b}$ est essentielle si l'on veut prévoir l'hydrodynamique en zone de surf. Dans les modèles de vague, qu'ils soient intégrés sur la période des vagues ou instationnaires (de type Boussinesq), $\mathrm{C}_{\mathrm{b}}$ est un paramètre clé. De récents travaux menés par les proposant (BONNETON, 2007) ont permis d'améliorer la paramétrisation de $\mathrm{C}_{\mathrm{b}}$ pour des vagues bidimensionnelles. Dans le cadre de cet atelier, seront étudiés à la fois l'impact d'une barre tridimensionnelle, mais aussi l'influence des forts courants de baïnes et du back-wash sur la cinématique des fronts d'ondes en zones de surf et de jet de rive. Pour évaluer la vitesse des vagues, plusieurs méthodes ont été déployées et notamment l'utilisation de lignes transversales de capteurs synchronisés (EPOC) composées d'un ADV et de deux capteurs de pression (figure 6b), testés en canal (Bretel et al., 2007). L'autre méthode mise en œuvre s'appuie sur l'exploitation des images vidéo: calcul de la vitesse des vagues à partir de cross- corrélation de timestacks (ALMAR et al., 2008, figure 6a) mais également développement de méthodes PIV sur des images instantanées 2D.

\subsection{Circulation générale et macro-structures tourbillonnaires}

De précédents travaux de modélisation (CASTELLE et al., 2006) ont permis de mettre en évidence d'une part la complexité de la courantologie au niveau des systèmes de barre-baïnes et d'autre part son rôle dans la compréhension de la morphodynamique des structures (construction, déformation......).

L'un des premiers objectifs consiste en l'établissement d'une carte $2 \mathrm{DH}$ de circulation moyenne de surface en se plaçant dans une hypothèse quasistationnaire. Dans un second temps, on s'attachera à étudier les instationnarités basses fréquences (de l'ordre de $10^{2}$ secondes) puis les instationnarités très basses fréquences (de l'ordre de $10^{3}$ secondes). Ceci nous conduit ensuite à étudier les macro structures tourbillonnaires horizontales. On pense que ces structures sont responsables d'une grande partie des échanges hydrodynamiques et sédimentaires 
entre la zone de surf et le large, à la fois en terme de mélange et de transport. On peut citer deux mécanismes principaux pour la génération de ces structures : les instabilités de cisaillement du courant de dérive («shear waves ») observées à Duck (OLTMAN-SHAY et al., 1989) et les courants sagittaux (« rip current») qui font l'objet de plus en plus d'études ces dernières années (parmi elles, TURNER et al, 2007 ; MAC MAHAN et al., 2006 ; BRANDER and SHORT, 2004) ou de campagnes spécifiques (RIPEX). Il existe à l'heure actuelle très peu d'expériences décrivant la dynamique de ces structures tourbillonnaires en milieu naturel. La description de cette dynamique dans les modèles actuels est donc très grossière. Enfin, un dernier point consistera à étudier la structure verticale des courants, en particulier au niveau des morphologies tridimensionnelles. Trois équipes, SHOM, EPOC et NPS se sont concentrés sur l'étude de cette circulation générale et macro-structures tourbillonnaires. Les méthodes employées ont consisté à déployer des instruments eulériens de type courantomètre électromagnétique ou acoustique mais également des profileurs verticaux (AWAC, ADCP) des profileurs horizontaux de courant (figure 7a, BRETEL et BONNETON, 2007) ainsi que des dériveurs lagrangiens (NPS, figure 7b).

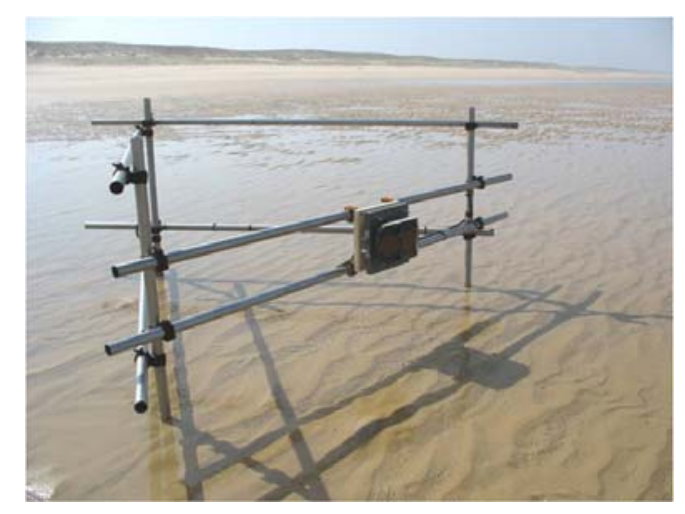

Figure 7a: HADCP déployé et testé par EPOC

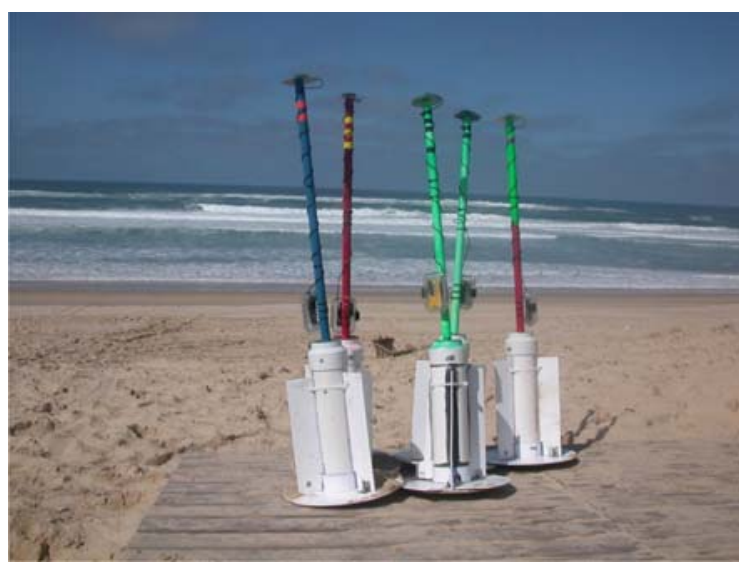

Figure $7 b$ : dériveurs lagrangiens déployés par la NPS

\section{4. forcages hydrodynamiques et réponses morphologiques pendant la campagne}

Dans cette partie nous rapporterons les premières observations faites pendant la campagne ECORS-Truc Vert 2008. La figure 8 représente les forçages hydrodynamiques pendant la campagne. Après une période calme en Février 2008 qui a permis le déploiement de trois instruments en zone subtidale ainsi qu'un levé très précis de la bathymétrie, la campagne a été marquée par une succession 
inhabituelle de 5 évènements très énergétiques associés à des hauteurs significatives de plus de $5 \mathrm{~m}$.
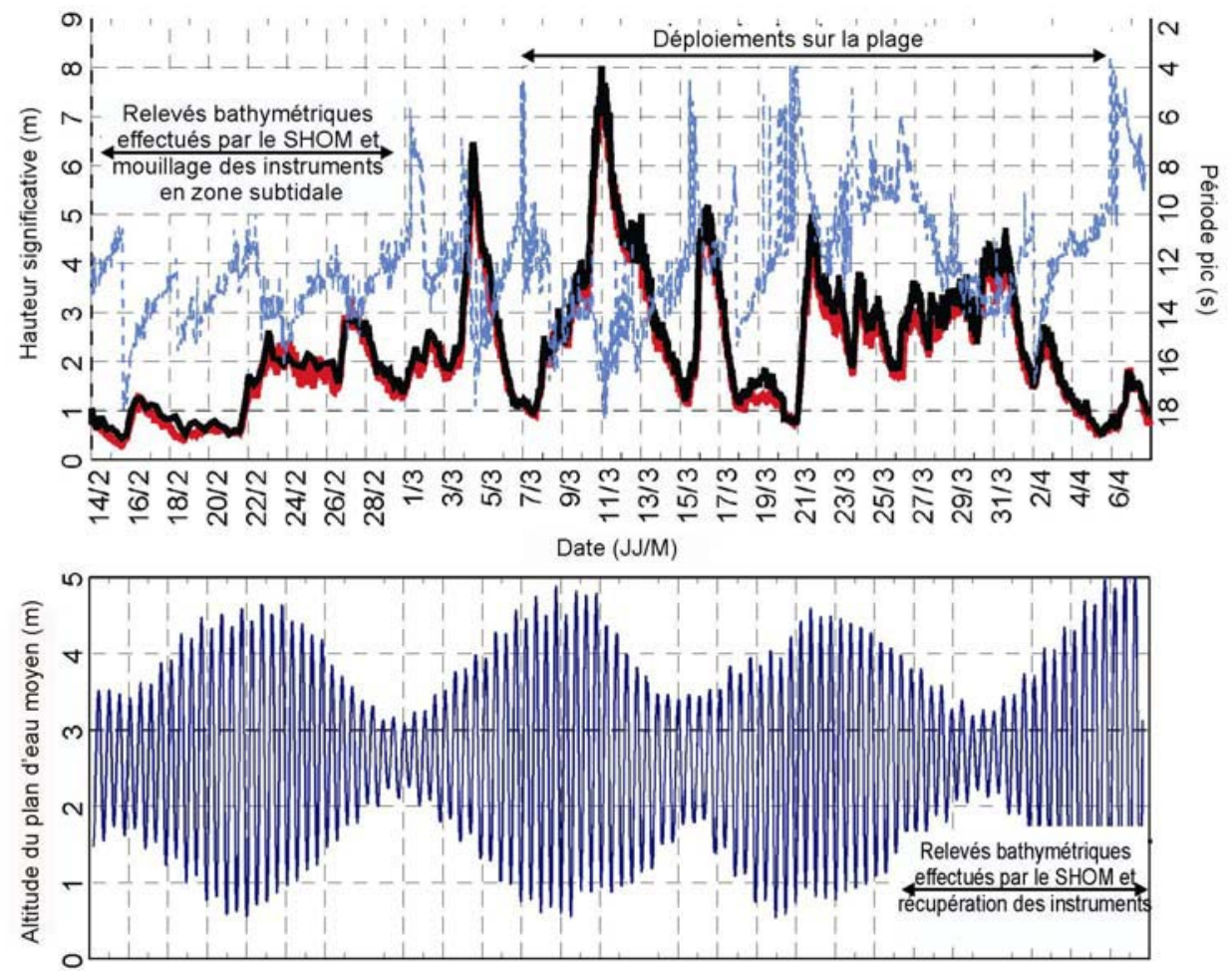

Figure 8: hauteur significative des vagues mesurées par $50 \mathrm{~m}$ (noire) et $20 \mathrm{~m}$ (rouge) et période pic (bleu pointillé) et altitude du plan d'eau (bleu continu).

4 de ces évènements ont pu être enregistrés par l'ensemble des instruments déployés en zone intertidale. En particulier, la tempête décennale du 11 mars 2008 associée à un marnage de près de $5 \mathrm{~m}$, des hauteurs significatives de plus de $8 \mathrm{~m}$ et des périodes pic de $18 \mathrm{~s}$ a généré des courants longitudinaux moyens supérieurs à $1.5 \mathrm{~m} / \mathrm{s}$. La barre interne a rapidement migré vers le sud de plusieurs dizaines de mètres recouvrant des instruments sous plusieurs mètres de sable. La barre externe très développée en début de campagne et qui présentait une forme en croissant (figure 9a) a migré de plusieurs dizaines de mètres vers le large et présentait une forme très linéaire après cet épisode. Les observations vidéo ont permis d'enregistrer des cycles de destruction/ construction/ migration des barres subtidales en croissant. Une migration vers le sud des barres externes de près de $350 \mathrm{~m}$ a été observée sur l'ensemble de la campagne. La figure $9 \mathrm{~b}$ représente la bathymétrie finale levée en Avril 2008. Un levé intermédiaire avait initialement été prévu mais n'a pu être réalisé à cause des conditions de vagues trop fortes. Ces observations uniques pour la côte aquitaine permettront de mieux comprendre la dynamique de ces structures en présence de fortes conditions de houle. 

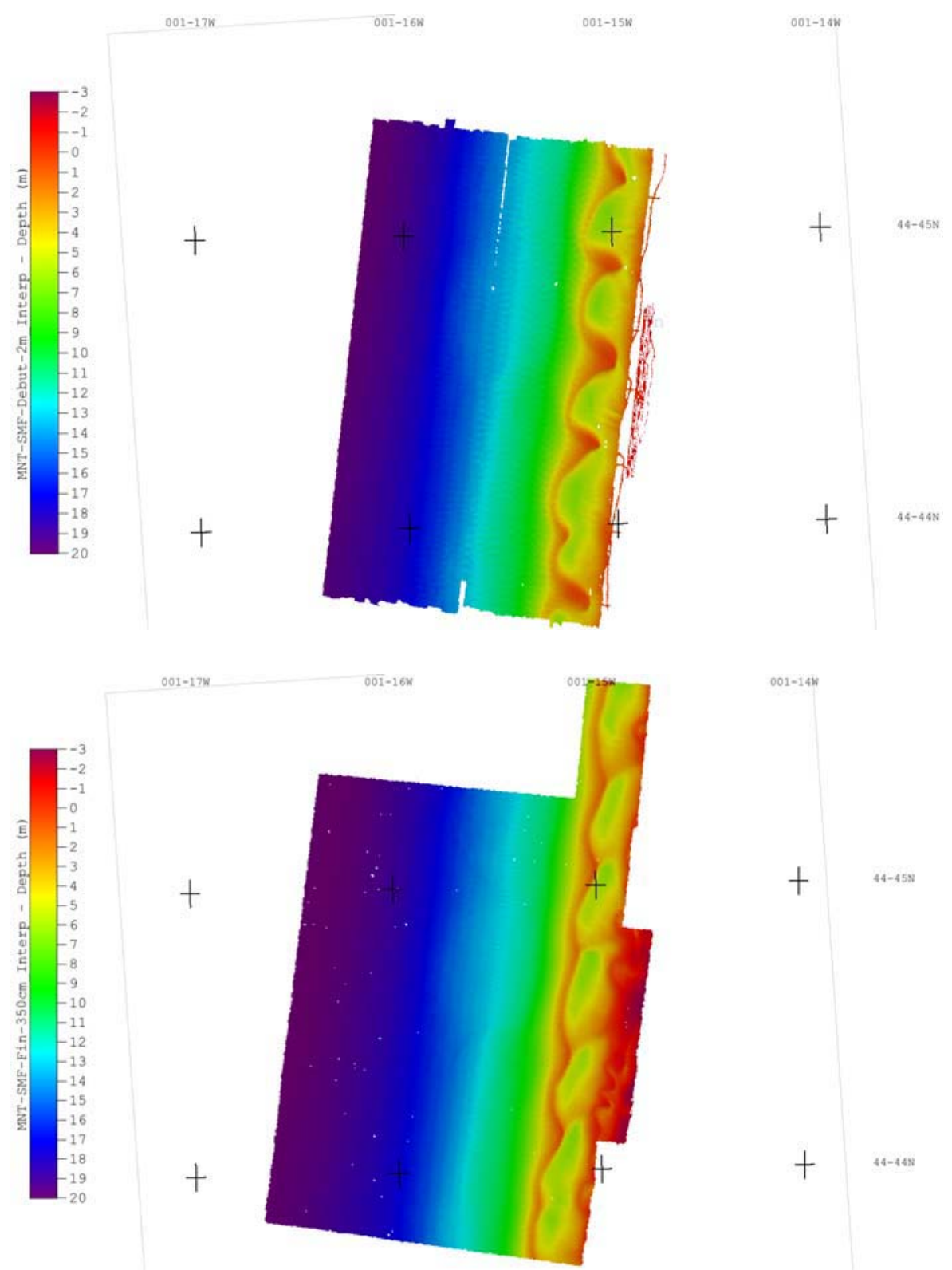

Figure 9 : relevé bathymétrique initial (Février 2008) et final (Avril 2008).

\section{Conclusions}

La campagne ECORS-Truc Vert 2008 a permis le travail en commun de plus de 100 personnes pendant près de 6 semaines avec l'objectif commun de mieux comprendre l'hydrodynamique et la morphodynamique des plages sableuses à barres. Elle a réuni des chercheurs de 15 laboratoires internationaux qui ont mesuré l'ensemble des processus impliqués dans la dynamique des plages : des micro processus de turbulence dans la couche limite jusqu'à l'étude des structures macro-tourbillonaires liées aux courants de refente. Cette campagne offre donc un 
jeu de données unique au monde aussi bien pour la compréhension des processus physiques et hydro-sédimentaires dans la zone de déferlement que pour l'amélioration et la validation des modèles numériques de houle et de morphodynamique, d'autant plus que les conditions de houle rencontrées pendant cette campagne ont été particulièrement énergétiques avec près de 6 évènements de tempêtes (Hs $>5 \mathrm{~m}$ ) en présence de mortes eaux (coefficient de 28) mais également en présence de vives eaux (coefficient de 109). Au-delà des données récoltées, la très bonne ambiance a facilité un échange important de savoir faire, en dépit des conditions météorologiques parfois difficiles. De nouvelles collaborations internationales devraient émerger dans les prochains mois. Cette campagne devrait ainsi contribuer à l'excellence de la recherche française en océanographie littorale.

\section{Références bibliographiques}

1 ALMAR R., BONNETON P., SENECHAL N., ROELVINK D. (2008). Wave celerity from video imaging:validation with field experiment on an Aquitanian beach. Accepté pour présentation à ICCE '08- Hamburg - Germany.

2 ALMAR R., SENECHAL N., COCO G. (2008). Estimation haute fréquence de la topographie intertidale d'une plage sableuse: application à la caractérisation des seuils d'engraissement et d'érosion. $\mathrm{X}^{\text {èmes }}$ Journées nationales Génie Civil-Génie Côtier, Sophia Antipolis.

3 BONJEAN D., FORAY P., PIEDRA-CUEVA I., MICHALLET H., BREUL P. HADDANI Y., MORY M., and ABADIE, S. (2004). Monitoring of the foundations of a coastal structure submitted to breaking waves : occurrence of momentary liquefaction. Proc. XIVth Int. Offshore and Polar Engineering Conf., Vol.II, ISOPE, pp 585-592.

4 BONNETON P. (2007). Modelling of periodic wave transformation in the inner surf zone. Ocean Engineering, 34 , pp1459-1471.

5 BRANDER S., SHORT A.D. (2004). Flow kinematics of a low-energy rip current system. J. of Coastal Research, 17:2, pp 468-481.

6 BRETEL P., PEREZ L., MOUAZÉ D. (2007). Mesure de la surface libre en zone de surf: validation en canal à houle d'un système de mesure in situ basé sur des capteurs de pression absolue. Présenté à l'ASF - Caen

7 BRETEL, P., BONNETON, N., (2007). HADCP 600KHz in the surf zone: currents study in a rip channel. Deployment. Limits. Promising first results ... but moorings to improve. ADCP In Action. San Diego. 
8 CASTELlE B., BONNETON P., DUPUIS H., SENECHAL N. (2007). Double bar beach dynamics on the high-energy meso-macrotidal French Aquitanian coast: a review. Marine Geology, 245, pp141-159.

9 CASTELLE B., BONNETON P., SENECHAL N., DUPUIS H., BUTEL D., MICHEL D. (2006). Dynamics of wave-induced currents over a multi-barred beach on the Aquitanian coast. Continental Shelf Research, 26, pp 113-131.

10 FROIDEFOND J.M., CASTAING P., JOUANNEAU J.M., PRUD'HOMME R., DINET A., (1993). Method for quantifying the total weight of suspended sediment at the surface of the sea from AVHRR-NOAA satellite data. Intern. Journ Remote Sensing., vol.14 (5), pp 885-894.

11 HURTHER D., U. LEMMIN (2003). Turbulent particle and momentum flux statistics in suspension flow. Water Resources Research, 39 (5).

12 HURTHER D. , MICHALLET H, GONDRAN X. (2007a). Turbulent measurements in the surf zone suspension. Journal of Coastal Research, SI50, pp 297-301.

13 HURTHER D., LEMMIN U., TERRAY E.A. (2007b). Turbulent transport in the outer region of rough wall open-channel flows : the contribution of large coherent shear stress structure (LC3S). Journal of Fluid Mechanics, 574, pp 465-493.

14 LAFON V., DUPUIS H., HOWA H., FROIDEFOND J.M. (2002a). Determining ridge and runnel longshore morphodynamic using SPOT imagery. Oceanologica Acta, 25(3-4), pp 149-158.

15 LAFON V., FROIDEFOND V., CASTAING P. (2002b). Spot shallow water bathymetry of a moderately tidal inlet based on field measurements. Remote Sensing of Envrionment, 81, pp 136-148.

16 MAGNE R., ARDHUIN F. (2008). Prévisions et rejeux des états de mer du globe à la plage. $\mathrm{X}^{\text {èmes }}$ Journées Nationales Génie Civil - Génie Côtier, Sophia Antipolis.

17 MACMAHAN J.H., THORNTON Ed B., RENIERS Ad J.H.M. (2006). Rip current review. Marine Geology, 53, pp 191-208.

18 MARTINY, N. (2002). Validation des corrections atmosphériques: application au capteur SeaWiFS en milieu côtier. Thèse, Université du Littoral Côte d'Opale, p. 231. 
19 MICHALLET, H., BARBIER-NEYRET, J.-P., MORY, M., \& PIEDRACUEVA, I. (2005). An optical system for monitoring seabed evolutions at a coastal structure. Proc. 5th Int. Conf. on Coastal Dynamics, ASCE, Barcelona (13p.).

20 MORY M., MICHALLET H., BONJEAN D., PIEDRA-CUEVA I., BARNOUD J.-M., FORAY P., ABADIE S., BREUL P. (2007). A field study of momentary liquefaction caused by waves around a coastal structure. ASCE Jounral of waterway, port, coastal, and Ocean Engineering, ISSN:0733-950X, 133(1), pp 28-38.

21 OLTMAN-SHAY J., HOWD J.A. and BIRKEMEIER W.A. (1989). Shear instabilities of the longshore current: a comparison of observations and computations. Journal of Geophysical Research, 94: pp 18,031-18,042.

22 PARISOT J.P., BUJAN S., BRETEL P., MICHEL D., LELABOUSSE C., GOURIOU T., SENECHAL N., CAPO S. (2007). Traitement des données GPS acquises sur le littoral aquitain: optimisation des mesures effectuées en quad. Présenté à l'ASF - Caen.

23 PIEDRA-CUEVA, I., MICHALLET, H., \& MORY, M. (2005). Wavelet analysis of pressure measurements near a coastal structure. Proc. 5th Int. Symp. on Ocean Wave Measurements and Analysis 'WAVES2005', ASCE, Madrid (11p.).

24 RAUBENHEIMER B., GUZA R.T., ELGAR S. (1996). Wave transformation accross the inner surf zone. J. Geophys. Res., 101, pp 25,58925,597 .

25 SENECHAL N., DUPUIS H., BONNETON P., HOWA H., PEDREROS R. (2001). Observation of irregular wave transformation in the surf zone over a gently sloping sandy beach on the French Atlantic coastline. Oceanologica Acta, 24 (-6), pp 545-556.

26 TURNER I.L., WHYTE D., RUESSINK B.G., RANASINGHE R. (2007). Observations of rip spacing, persistence and mobility at a long straight coastline. Marine Geology, 236, pp 209-221.

Nous remercions toutes les personnes, institutions, collectivités, entreprises et organismes qui ont contribué à la réussite de ce projet, en particulier le Projet ECORS (SHOM-DGA) pour le soutien financier, matériel et humain.

Cette campagne est dédiée à Denis Michel, Maître de Conférences à l'Université Bordeaux I, chercheur à l'UMR EPOC, décédé le 16 juin 2007 à Biscarrosse lors d'une campagne de préparation à la campagne ECORS-Truc Vert 2008. 